\title{
The First Cohomology Groups of Infinite Dimensional Lie Algebras ${ }^{11}$
}

\author{
By \\ Nobutada NAKANISHI*
}

\section{Introduction}

Let $V$ be a finite dimensional vector space. We denote by $D(V)$ the Lie algebra consisting of all formal vector fields over $V$. Let $L$ be a Lie subalgebra of $D(V)$. We are interested in the first cohomology group $H^{1}(L)$ of a Lie algebra $L$ with adjoint representation.

Let $L$ be an infinite dimensional transitive simple Lie algebra, that is, $L$ is one of $D(V), L_{\overline{3}\{}, L_{\overline{3} \mathfrak{p}}$, or $L_{\mathrm{ct}}$. (For a notation, see $\S 2$.) It is known in $\mathrm{T}$. Morimoto [5] that $H^{1}(D(V))=H^{1}\left(L_{\mathrm{ct}}\right)=0$, and $\operatorname{dim} H^{1}\left(L_{\mathfrak{z} \mathfrak{l}}\right)=\operatorname{dim} H^{1}\left(L_{\mathfrak{z} \mathfrak{p}}\right)=1$.

In this paper we will treat the following two types of infinite dimensional Lie algebras:

(1) Infinite dimensional transitive graded Lie algebras $\mathfrak{g}=\sum_{p=-1}^{\infty} \mathfrak{g}_{p}$. (For a precise definition, see $\S 1$.)

(2) Infinite dimensional intransitive Lie algebras $L\left[W^{*}\right]$ whose transitive parts $L$ are infinite and simple. (In this case $W$ is a subspace of $V$.)

In Section 3 and Section 4, we will give two criteria for $H^{1}(\mathfrak{g})$ to be of finite dimension. More precisely we will prove

Theorem A. Let $\mathfrak{g}=\sum_{p=-1}^{\infty} \mathfrak{g}_{p}$ be an infinite transitive graded Lie algebra with a semi-simple linear isotropy algebra $\mathfrak{g}_{0}$. Then $H^{1}(\mathfrak{g})$ is finite dimensional.

Theorem B. Let $\mathfrak{g}=\sum_{p=-1}^{\infty} \mathfrak{g}_{p}$ be an infinite transitive graded Lie algebra whose linear isotropy algebra $\mathfrak{g}_{0}$ contains an element $e$ which satisfies $\left[e, x_{p}\right]=$

Communicated by N. Shimada, February 8, 1980.

* Department Mathematics, Maizuru Technical College, Maizuru 625, Japan.

1) This research was partially supported by Grant-in-Aid for Scientific Research (No. 474043), Ministry of Education. 
$p x_{p}$ for all $x_{p} \in \mathfrak{g}_{p}$. Then $H^{1}(\mathfrak{g})$ is finite dimensional. Furthermore if $\mathfrak{g}$ is derived from $\mathfrak{g}_{0}$, then $H^{1}(\mathfrak{g})$ is isomorphic to $n\left(g_{0}\right) / \mathfrak{g}_{0}$, where $n\left(\mathfrak{g}_{0}\right)$ denotes the normalizer of $\mathfrak{g}_{0}$ in $\mathfrak{g l}\left(\mathfrak{g}_{-1}\right)$.

It may well be doubted if every infinite transitive graded Lie algebra $g$ has the finite dimensional cohomology group $H^{1}(\mathfrak{g})$. But unfortunately this presumption is false. In Section 5 we will give an easy condition for $g$ to be $\operatorname{dim} H^{1}(\mathfrak{g})=\infty$. (For such a Lie algebra $\mathfrak{g}$, we can construct derivations of arbitrarily large negative degree.)

That is, we will prove

Theorem $\mathbb{C}_{\text {. Let }} \mathrm{g}=\sum_{p=-1}^{\infty} \mathfrak{g}_{p}$ be an infinite transitive graded Lie algebra which satisfies $\mathfrak{g}^{(2)}=\left[\mathfrak{g}^{(1)}, \mathfrak{g}^{(1)}\right]=0$, where $\mathfrak{g}^{(1)}=[\mathfrak{g}, \mathfrak{g}]$. Then $H^{1}(\mathfrak{g})$ is infinite dimensional.

In Section 6 our objects are infinite intransitive Lie algebras $L\left[W^{*}\right]$. Let $V=U+W$ (direct sum). We denote by $S\left(W^{*}\right)$ the ring of formal power series over $W$. Let $L$ be an infinite transitive simple Lie algebra over $U$. Then a Lie algebra $L\left[W^{*}\right]$ is obtained as a topological completion of $L \otimes S\left(W^{*}\right)$. These Lie algebras $L\left[W^{*}\right]$ are obtained as the result of the classification theorem of infinite intransitive Lie algebras [6]. In determining $H^{1}\left(L\left[W^{*}\right]\right)$, V. Guillemin's work is essential. Using his results we will prove

Theorem $\mathbb{D}$. Let $D(W)$ be a Lie algebra of all formal vector fields over $W$ and let e be a basis of one dimensional center of $\mathrm{gl}(U)$. Then we have

$H^{1}\left(L\left[W^{*}\right]\right) \cong\left\{\begin{array}{l}D(W) \quad \text { for } L=D(U) \text { or } L_{\mathrm{ct}}(U), \\ D(W)+S\left(W^{*}\right) \otimes e \quad \text { for } L=L_{\mathfrak{\jmath} \mathfrak{l}}(U) \text { or } L_{\mathfrak{\xi} p}(U) .\end{array}\right.$

Above results can be considered as a formal version of Y. Kanie [3]. In a forthcoming paper, we will give an example of an infinite intransitive Lie algebra $L$ such that $H^{1}(L)=0$.

Throughout this paper, all vector spaces and Lie algebras are assumed to be defined over the field $\mathbb{C}$ of complex numbers.

\section{§1. Infinite Transitive Graded Lie Algebras}

In this section, we define transitive graded Lie algebras which we will study in the subsequent sections. 
Defimition 1.1. Let $g$ be a Lie algebra. Assume that there is given a family $\left\{\mathfrak{g}_{p}\right\}_{p \geqq-1}$ of subspaces of $\mathfrak{g}$ which satisfies the following conditions:

a) $\mathfrak{g}=\sum_{p=-1}^{\infty} \mathfrak{g}_{p} \quad$ (direct sum);

b) $\operatorname{dim} \mathfrak{g}_{p}<\infty$;

c) $\left[\mathfrak{g}_{p}, \mathfrak{g}_{q}\right] \subset \mathfrak{g}_{p+q}$;

d) For every non-zero $x_{p} \in \mathfrak{g}_{p}, p \geqq 0$, there is an element $x_{-1} \in \mathfrak{g}_{-1}$ such that $\left[x_{p}, x_{-1}\right] \neq 0$. Under these conditions, we say that the direct sum $g$ $=\sum_{p=-1}^{\infty} \mathfrak{g}_{p}$ or simply $\mathfrak{g}$ is a transitive graded Lie algebra.

By conditions c) and d), $\mathfrak{g}_{0}$ is considered as a Lie subalgebra of $\mathfrak{g l}\left(\mathfrak{g}_{-1}\right)$. The Lie algebra $\mathfrak{g}_{0}$ is called the linear isotropy algebra of $\mathfrak{g}$. A graded Lie algebra $\mathfrak{g}$ is said to be irreducible if the representation of $\mathfrak{g}_{0}$ on the vector space $\mathfrak{g}_{-1}$ given by $\left[\mathfrak{g}_{0}, \mathfrak{g}_{-1}\right] \subset \mathfrak{g}_{-1}$ is irreducible.

Definition 1.2. The space $\mathfrak{g}_{0}^{(p)}$ which is called the $p$-th prolongation of $\mathfrak{g}_{0}$ is defined by

$$
\mathfrak{g}_{0}^{(p)}=\mathfrak{g}_{0} \otimes S^{p}\left(\mathfrak{g}_{-1}^{*}\right) \cap \mathfrak{g}_{-1} \otimes S^{p+1}\left(\mathfrak{g}_{-1}^{*}\right),
$$

where $S^{p}\left(g_{-1}^{*}\right)$ denotes the $p$-times symmetric tensor of the dual space $g_{-1}^{*}$ of $\mathfrak{g}_{-1}$.

We say that $\mathfrak{g}_{0}$ is of finite type if $\mathfrak{g}_{0}^{(p)}=0$ for some (and hence for all larger) p. Otherwise we say that $\mathfrak{g}_{0}$ is of infinite type. Put $\mathfrak{g}_{0}^{(-1)}=\mathfrak{g}_{-1}, \mathfrak{g}_{0}^{(0)}=\mathfrak{g}_{0}$ and $\tilde{\mathfrak{g}}=\sum_{p=-1}^{\infty} \mathfrak{g}_{0}^{(p)}$. Then $\tilde{\mathfrak{g}}$ has a Lie algebra structure with respect to a canonical bracket operation. We say that the transitive graded Lie algebra $\tilde{\mathfrak{g}}=\sum_{p=-1}^{\infty} \mathfrak{g}_{0}^{(p)}$ thus obtained is derived from $\mathfrak{g}_{0}$. If $\mathfrak{g}$ is an abstract transitive graded Lie algebra with a linear isotropy algebra $\mathfrak{g}_{0}$, then $\mathfrak{g}$ is considered as a graded Lie subalgebra of $\tilde{\mathfrak{g}}$. It is clear that if a transitive graded Lie algebra $\mathfrak{g}$ is of infinite dimension, its linear isotropy algebra $g_{0}$ must be of infinite type.

Let $A$ be a Lie algebra. A derivation $c$ of $A$ is a linear mapping from $A$ to itself satisfying $c[x, y]=[c(x), y]+[x, c(y)]$ for all $x, y \in A$. We denote by $\operatorname{Der}(A)$ (resp. ad $(A)$ ) the derivation algebra (resp. the algebra of inner derivations of $A$ ). Then, by definition, the first cohomology group $H^{1}(A)$ of $A$ with adjoint representation is equal to the space $\operatorname{Der}(A) / \operatorname{ad}(A)$. A derivation $c$ of a graded Lie algebra $\mathfrak{g}=\sum_{p=-1}^{\infty} \mathfrak{g}_{p}$ is said to be of degree $r$ or deg $c=r$ if it satisfies $c\left(\mathfrak{g}_{p}\right) \subset \mathfrak{g}_{p+r}$ for all $p$. 


\section{§2. Infinite Transitive Simple Lie Algebras}

It is well-known that there are the following four classes of infinite transitive simple Lie algebras over $\boldsymbol{C}$ (see [5]).

(1) $L_{\mathrm{gl}}(n)$ : the Lie algebra of all formal (or better, formal power series) vector fields in $n$-variables $x_{1}, x_{2}, \ldots, x_{n}$.

(2) $L_{\mathfrak{3} \mathfrak{l}}(n)$ : the Lie algebra of formal vector fields in $n$-variables $x_{1}$, $x_{2}, \ldots, x_{n}$, preserving the volume form $d x_{1} \wedge d x_{2} \wedge \cdots \wedge d x_{n}$.

(3) $L_{\overline{5} p}(2 n)$ : the Lie algebra of formal vector fields in $2 n$-variables $x_{1}$, $x_{2}, \ldots, x_{n}, y_{1}, y_{2}, \ldots, y_{n}$, preserving the symplectic form $\sum_{i=1}^{n} d x_{i} \wedge d y_{i}$.

(4) $L_{\mathrm{ct}}(2 n+1)$ : the Lie algebra of formal vector fields in $(2 n+1)$-variables $z, x_{1}, x_{2}, \ldots, x_{n}, y_{1}, y_{2}, \ldots, y_{n}$, preserving the contact form $d z+2^{-1} \sum_{i=1}^{n} x_{i}$. $d y_{i}-y_{i} d x_{i}$, up to functional factors.

We will often write $D(V)$ for $L_{\mathrm{gI}}(n)$, where $V$ is an $n$-dimensional vector space with a basis $\partial / \partial x_{1}, \ldots, \partial / \partial x_{n}$. Let $L$ be one of Lie algebras $D(V), L_{31}$ and $L_{\text {₹p }}$. Each $L$ has the natural filtration $\left\{L_{p}\right\}_{p \in Z}$ defined as follows.

$$
\begin{aligned}
& L_{p}=L \quad \text { for } \quad p \leqq-1 ; \\
& L_{0}=\{X \in L ; \text { the value } X(0) \text { of } X \text { at the origin }=0\} ; \\
& L_{p}=\left\{X \in L_{p-1} ; \quad[X, L] \subset L_{p-1}\right\} \quad \text { for } p \geqq 1 .
\end{aligned}
$$

Then the decreasing sequence of subspaces: $L=L_{-1} \supset L_{0} \supset L_{1} \supset L_{2} \supset \cdots$ satisfies

(a) $\bigcap_{p=-1}^{\infty} L_{p}=0$;

(b) $\left[L_{p}, L_{q}\right] \subset L_{p+q}$;

(c) $\operatorname{dim} L_{p} / L_{p+1}<\infty$.

Put $\mathfrak{g}_{p}(L)=L_{p} / L_{p+1}$. Then by (b), (c) and the definition of $L_{p}, p \geqq 1$, we have the transitive graded Lie algebra $\operatorname{gr}(L)=\sum_{p=-1}^{\infty} \mathfrak{g}_{p}(L)$. We also have the Lie algebra $L^{\prime}=\prod_{p=-1}^{\infty} \mathfrak{g}_{p}(L)$, which is the completion of $\operatorname{gr}(L)$.

Under these notations we will summarize a few useful properties of $L$. (For the proof, see Kobayashi-Nagano [4].)

(1) Each $L$ is an infinite transitive irreducible Lie algebra and moreover $L$ is isomorphic to $L$ ', where the word "irreducible" means that the action of $\mathfrak{g}_{0}(L)$ on $\mathfrak{g}_{-1}(L)$ is irreducible.

(2) The linear isotropy algebras $\mathfrak{g}_{0}(L)$ of $D(V), L_{\mathfrak{\xi l} \text { I }}$ and $L_{\mathfrak{\xi} \mathfrak{p}}$ are $\mathfrak{g l}(n, \boldsymbol{C})$, 
$\mathfrak{s l}(n, \mathbb{C})$ and $\mathfrak{s p}(n, \mathbb{G})$ respectively, and for $p \geqq 1 \mathfrak{g}_{p}(L)$ is isomorphic to the $p$-th prolongation $\mathfrak{g}_{0}(L)^{(p)}$ of $\mathfrak{g}_{0}(L)$.

(3) For $\mathfrak{g}_{0}(L)=\mathfrak{s l}(n, \boldsymbol{C})$ or $\mathfrak{s p}(n, \boldsymbol{C})$, it holds that

(i) $\left[\mathfrak{g}_{0}(L)^{(r)}, \mathfrak{g}_{0}(L)^{(s)}\right]=\mathfrak{g}_{0}(L)^{(r+s)} \quad$ for $\quad r, s \geqq 0$,

(ii) $\mathfrak{g}_{0}(L)$ acts irreducibly on $\mathfrak{g}_{0}(L)^{(r)}$ for $r \geqq-1$.

By the classification theorem of Kobayashi-Nagano [4], we know that there are only three classes of transitive simple irreducible Lie algebras of infinite type over $\boldsymbol{C}$, that is, they are $D(V), L_{\mathfrak{s} \mathfrak{l}}$ and $L_{\mathfrak{s} \mathfrak{p}}$.

For the contact Lie algebra $L_{\mathrm{ct}}(2 n+1)$ (or simply $L_{\mathrm{ct}}$ ), we must define another filtration.

$$
\begin{aligned}
& L_{p}=L_{\mathrm{ct}} \quad \text { for } \quad p \leqq-2 ; \\
& L_{-1}=\left\{X \in L_{\mathrm{ct}} ;\langle X, \theta\rangle_{0}=0, \text { where } \theta \text { is the contact form }\right\} ; \\
& L_{0}=\left\{X \in L_{\mathrm{ct}} ; X(0)=0\right\} ; \\
& L_{p}=\left\{X \in L_{p-1} ;\left[X, L_{-1}\right] \subset L_{p-1}\right\} \quad \text { for } \quad p \geqq 1 .
\end{aligned}
$$

Using this filtration, $L_{\mathrm{ct}}$ is isomorphic to $\prod_{p=-2}^{\infty} \mathfrak{g}_{p}(L)$. For the subsequent discussion about $L_{\mathrm{ct}}$, we have only to recall that

(4) $L_{-1}=\left[L_{\mathrm{ct}}, L_{1}\right]$

In Section 3 and Section 6, we essentially use the following facts which were proved by T. Morimoto [5].

Theorem 2.1. Let $L$ be an infinite transitive simple Lie algebra over $\mathbb{C}$. Then

$$
H^{1}(L) \cong \begin{cases}0 & \text { for } L=D(V) \text { or } L_{\mathrm{ct}} \\ C & \text { for } L=L_{\hat{g} \mathfrak{l}} \text { or } L_{\hat{\mathfrak{g}} \mathfrak{p}}\end{cases}
$$

Remark 1. Let $L$ be one of Lie algebras $L_{\mathfrak{3} \mathfrak{l}}$ or $L_{\mathfrak{\xi} \mathfrak{p}}$. Since $L$ is isomorphic with the Lie algebra $L^{\prime}=\prod_{p=-1}^{\infty} \mathfrak{g}_{p}(L)$, their isotropy algebras $\mathfrak{s}(V)$ and $\mathfrak{s p}(V)$ are considered to be subalgebras of them. Let $e$ denote a unit matrix in $\operatorname{gl}(V)$. Then the above theorem asserts that ad $(e)$ yields a basis of one dimensional space $H^{1}(L)$.

Remark 2. Let $\mathfrak{g r}(L)$ be a graded Lie algebra associated with an infinite transitive simple Lie algebra $L$. Then we also have $H^{1}(\mathfrak{g r}(L))=0$ for $L=D(V)$ or $L_{\mathrm{ct}}$, and $H^{1}(\mathfrak{g r}(L)) \cong \boldsymbol{C}$ for $L=L_{\overline{\mathrm{z}} \mathfrak{l}}$ or $L_{\mathfrak{z} \mathfrak{p}}$. These facts are particularly used in Section 3. 


\section{§3. The First Cohomology Groups of Infinite Transitive Graded Lie Algebras

Throughout this section, let $\mathfrak{g}=\sum_{p=-1}^{\infty} \mathfrak{g}_{p}$ be an infinite (dimensional) transitive graded Lie algebra over $\mathbb{C}$ and let its linear isotropy algebra $\mathfrak{g}_{0}$ be semi-simple. Put $\mathfrak{g}_{-1}=V$. Then $\mathfrak{g}_{0}$ is considered as a Lie subalgebra of $\operatorname{gl}(V)$. First we will determine the type of $\mathfrak{g}$.

Since $\mathrm{g}_{0}$ is semi-simple, we can decompose $V$ into $V=V_{1}+V_{2}+\cdots+V_{k}$ (vector space direct sum), where each $V_{i}(i=1,2, \ldots, k)$ is a $g_{0}$-invariant subspace and $\mathfrak{g}_{0}$ acts irreducibly on $V_{i}$. We denote by $\mathfrak{h}_{i}$ the Lie algebra of linear transformations of $V_{i}$ induced by $\mathfrak{g}_{0}$. By the natural inclusion, $\mathfrak{h}_{i}$ is considered as a Lie subalgebra of $\operatorname{gl}(V)$. We also denote it by the same letter $\mathfrak{h}_{i}$ if there is no confusion. Put $\mathfrak{n}_{i}=\left\{t \in \mathfrak{g}_{0} ; t\left(V_{j}\right)=0\right.$ for all $\left.j \neq i\right\}$. Then each $\mathfrak{n}_{i}$ is an ideal of $\mathfrak{g}_{0}$ and $n_{1}+\cdots+n_{k}$ is a direct sum as Lie algebras. It clearly holds

$$
\mathrm{n}_{1}+\cdots+\mathfrak{n}_{k} \subset \mathfrak{g}_{0} \subset \mathfrak{h}_{1}+\cdots+\mathfrak{h}_{k} .
$$

Lemma 3.1. For $p \geqq 1, \mathfrak{g}_{0}^{(p)}=\mathfrak{n}_{1}^{(p)}+\cdots+\mathfrak{n}_{k}^{(p)}$ (direct sum).

Proof. Let $t: V \times \cdots \times V \rightarrow V$ be an element of $g_{0}^{(p)}$. First note that $t\left(v_{1}, \ldots\right.$, $\left.v_{p+1}\right)=0$ if $v_{i} \in V_{i}, v_{j} \in V_{j}$ for $i \neq j$. (It is easy to see that $t\left(v_{1}, \ldots, v_{p+1}\right) \in V_{i} \cap V_{j}$.) Let $v_{1}, \ldots, v_{p+1} \in V$ and $v_{i}=v_{i}^{1}+\cdots+v_{i}^{k}$ with $v_{i}^{1} \in V_{1}, v_{i}^{2} \in V_{2}, \ldots, v_{i}^{k} \in V_{k}$ for $i=1, \ldots, p+1$. Then by the above remark, we have

$$
\begin{aligned}
t\left(v_{1}, \ldots, v_{p+1}\right) & =t\left(v_{1}^{1}, \ldots, v_{p+1}^{1}\right)+\cdots+t\left(v_{1}^{k}, \ldots, v_{p+1}^{k}\right) \\
& =t_{1}\left(v_{1}^{1}, \ldots, v_{p+1}^{1}\right)+\cdots+t_{k}\left(v_{1}^{k}, \ldots, v_{p+1}^{k}\right) \\
& =t_{1}\left(v_{1}, \ldots, v_{p+1}\right)+\cdots+t_{k}\left(v_{1}, \ldots, v_{p+1}\right),
\end{aligned}
$$

where $t_{i}$ denotes an element of $\mathfrak{n}_{i}^{(p)}$ induced by $t$. (Since $t_{i}\left(*, v_{1}^{\prime}, \ldots, v_{p}^{\prime}\right) \in \mathfrak{n}_{i}$ for $v_{1}^{\prime}, \ldots, v_{p}^{\prime} \in V, t_{i}$ is an element of $\mathfrak{n}_{i}^{(p)}$.) Since $\mathfrak{n}_{1}+\cdots+\mathfrak{n}_{k}$ is a direct sum, our assertion is obvious.

q.e.d.

Since $\mathfrak{g}$ is infinite dimensional and $\mathfrak{g}_{p}$ is a subspace of $\mathfrak{g}_{0}^{(p)}, \mathfrak{g}_{0}$ must be of infinite type by Lemma 3.1. From now on, without loss of generality, we assume that $\mathfrak{n}_{1}, \ldots, \mathfrak{n}_{l}(l \leqq k)$ are of infinite type and $\mathfrak{n}_{l+1}, \ldots, \mathfrak{n}_{k}$ are of finite type.

Lemma 3.2. Let $\mathrm{g}_{0}$ be a linear isotropy algebra of an infinite transitive graded Lie algebra $\mathfrak{g}$. Then there exists a Lie subalgebra $\mathfrak{g}_{b}$ of finite type of $\mathfrak{h}_{l+1}+\cdots+\mathfrak{h}_{k}$ and $\mathfrak{g}_{0}$ is written as 


$$
\left.\mathfrak{g}_{0}=\mathfrak{n}_{1}+\cdots+\mathfrak{n}_{l}+\mathfrak{g}_{b} \quad \text { (Lie algebra direct sum }\right),
$$

where each ideal $\mathfrak{n}_{i}(i=1, \ldots, l)$ is isomorphic to either $\mathfrak{s l}\left(V_{i}\right)$ or $\mathfrak{s p}\left(V_{i}\right)$.

Proof. Let $\pi: \mathfrak{g}_{0} \rightarrow \mathfrak{h}_{i}(i=1, \ldots, l)$ be a natural projection. Since $\pi$ is a Lie algebra homomorphism, $\mathfrak{g}_{0} / \operatorname{Ker} \pi$ is isomorphic to $\mathfrak{h}_{i}$. Recall that the quotient space of a semi-simple Lie algebra is also semi-simple. Thus $\mathfrak{h}_{i}$ is semisimple and its center is zero. Moreover each $\mathfrak{h}_{i}$ acts irreducibly on $V_{i}$ and is of infinite type. Hence by the classification theorem of transitive irreducible Lie algebras of infinite type, we know that $\mathfrak{h}_{\imath}$ must be equal to either $\mathfrak{s l}\left(V_{i}\right)$ or $\mathfrak{s p}\left(V_{i}\right)$. Since $\mathfrak{n}_{i}$ is an ideal of $\mathfrak{h}_{i}$, we have $\mathfrak{n}_{i}=\mathfrak{s l}\left(V_{i}\right)$ or $\mathfrak{s p}\left(V_{i}\right)$. $\left(\mathfrak{s l}\left(V_{i}\right)\right.$ and $\mathfrak{s p}\left(V_{i}\right)$ are naturally imbedded in $\mathfrak{g l}(V)$.) Note that $\mathfrak{n}_{1}=\mathfrak{h}_{1}, \mathfrak{n}_{2}=\mathfrak{h}_{2}, \ldots, \mathfrak{n}_{l}=\mathfrak{h}_{l}$. Then we can find a subspace $\mathfrak{g}_{b}$ such that $\mathfrak{n}_{l+1}+\cdots+\mathfrak{n}_{k} \subset \mathfrak{g}_{b} \subset \mathfrak{h}_{l+1}+\cdots+\mathfrak{h}_{k}$. Considering (3.1), we obtain the expression of $g_{0}$ as (3.3). By Lemma 3.1, we also have $\mathfrak{g}_{b}^{(p)}=\mathfrak{n}_{l+1}^{(p)}+\cdots+\mathfrak{n}_{k}^{(p)}$. Thus $\mathfrak{g}_{b}$ is of finite type.

q.e.d.

Next we will determine the type of $g_{1}$. From (3.3) in Lemma 3.2, we have $\mathfrak{g}_{0}^{(1)}=\mathfrak{n}_{1}^{(1)}+\cdots+\mathfrak{n}_{l}^{(1)}+\mathfrak{g}_{b}^{(1)}$, and $\mathfrak{g}_{1}$ is a subspace of $\mathfrak{g}_{0}^{(1)}$. Without loss of generality, we assume that $\mathfrak{g}_{1} \cap \mathfrak{n}_{1}^{(1)} \neq 0, \ldots, \mathfrak{g}_{1} \cap \mathfrak{n}_{m}^{(1)} \neq 0$ and $\mathfrak{g}_{1} \cap \mathfrak{n}_{m+1}^{(1)}=0, \ldots, \mathfrak{g}_{1} \cap \mathfrak{n}_{l}^{(1)}$ $=0$. Then we have

Lemma 3.3. $\mathfrak{g}_{1}$ has the following form:

$$
\mathfrak{g}_{1}=\mathfrak{n}_{1}^{(1)}+\cdots+\mathfrak{n}_{m}^{(1)}+H_{1},
$$

where $H_{1}$ is a subspace of $\mathfrak{g}_{b}^{(1)}$.

Proof. For $i=1, \ldots, m, \mathfrak{g}_{1} \cap \mathfrak{n}_{i}^{(1)}$ is an $n_{i}$-invariant subspace of $\mathfrak{n}_{i}^{(1)}$. By the property (3) (ii) in Section 2, we have $\mathfrak{g}_{1} \supset \mathfrak{n}_{1}^{(1)}+\cdots+\mathfrak{n}_{m}^{(1)}$. Hence there exists a subspace $H_{1}$ of $\mathfrak{n}_{m+1}^{(1)}+\cdots+\mathfrak{n}_{l}^{(1)}+\mathfrak{g}_{b}^{(1)}$ such that $\mathfrak{g}_{1}=\mathfrak{n}_{1}^{(1)}+\cdots+\mathfrak{n}_{m}^{(1)}+H_{1}$ and $H_{1} \cap \mathfrak{n}_{m+1}^{(1)}=0, \ldots, H_{1} \cap \mathfrak{n}_{l}^{(1)}=0$. For $j=m+1, \ldots, l$, decompose $t \in H_{1}$ into $t=t_{m+1}+\cdots+t_{l}+t_{b}$ with $t_{m+1} \in \mathfrak{n}_{m+1}^{(1)}, \ldots, t_{l} \in \mathfrak{n}_{l}^{(1)}, t_{b} \in \mathfrak{g}_{b}^{(1)}$. Define a subspace $A_{j}$ of $\mathrm{n}_{j}^{(1)}$ by

$$
A_{j}=\left\{t_{j} \in \mathfrak{n}_{j}^{(1)} ; t=t_{m+1}+\cdots+t_{l}+t_{b} \in H_{1}\right\} .
$$

For all $x_{j} \in \mathfrak{n}_{j}$ and $t_{j} \in A_{j}$, it holds that $\left[x_{j}, t_{j}\right]=\left[x_{j}, t\right] \in \mathfrak{n}_{j}^{(1)} \cap \mathfrak{g}_{l}=\{0\}$. This means that $\left[\mathrm{n}_{j}, A_{j}\right]=0$. Using the property (3) (ii), we have $A_{j}=0$ for $j$ $=m+1, \ldots, l$. Hence $H_{1} \subset \mathfrak{g}_{b}^{(1)}$.

q.e.d.

Since $\mathfrak{g}_{p} \supset\left[\mathfrak{g}_{1},\left[\mathfrak{g}_{1},\left[\ldots,\left[\mathfrak{g}_{1}, \mathfrak{g}_{1}\right] \ldots\right]\right.\right.$ for $p>1, \mathfrak{g}_{p}$ contains $\mathfrak{n}_{1}^{(p)}+\cdots+\mathfrak{n}_{m}^{(p)}$ by Lemma 3.3 and the property (3) (i) in Section 2. By the same argument as Lemma 3.3, we get 
Lemma 3.4. For $p>1, \mathfrak{g}_{p}$ has the following form:

$$
\mathrm{g}_{p}=\mathrm{n}_{1}^{(p)}+\cdots+\mathrm{n}_{m}^{(p)}+H_{p},
$$

where $H_{p}$ is a subspace of $\mathfrak{g}_{b}^{(p)}$. (For sufficiently large $p, H_{p}=0$ since $\mathfrak{g}_{b}$ is of finite type.)

By Lemma 3.3 and Lemma 3.4, we can easily determine the form of the given infinite transitive graded Lie algebra $\mathfrak{g}=\sum_{p=-1}^{\infty} \mathfrak{g}_{p}$. That is, we have

Proposition 3.5. Let $\mathfrak{g}=\sum_{p=-1}^{\infty} \mathfrak{g}_{p}$ be an infinite transitive graded Lie algebra. Then $\mathrm{g}$ has the following form:

$$
\mathfrak{g}=G_{1}+\cdots+G_{m}+G_{m+1}^{\prime}+\cdots+G_{l}^{\prime}+G_{b}^{\prime} \quad(\text { direct sum }),
$$

where $G_{i}(i=1, \ldots, m)$ is of the form $\mathfrak{g r}\left(L_{\mathfrak{z} \mathfrak{l}}\left(V_{i}\right)\right)$ or $\mathfrak{g r}\left(L_{\bar{\beta} \mathfrak{p}}\left(V_{\imath}\right)\right)$, and $G_{j}^{\prime}(j$ $=m+1, \ldots, l)$ is of the form $V_{j}+\mathfrak{s l}\left(V_{j}\right)$ or $V_{j}+\mathfrak{s p}\left(V_{j}\right)$, and $G_{b}^{\prime}$ is a finite dimensional Lie algebra. (From now on, we put $G^{\prime}=G_{m+1}^{\prime}+\cdots+G_{l}^{\prime}+G_{b}^{\prime}$. Then $G^{\prime}$ is a finite dimensional ideal of $\mathfrak{g}$.)

For computing $H^{1}(\mathfrak{g})$, we need some lemmas.

Lemma 3.6. Let $A$ be an abstract Lie algebra and let $A_{i}(i=1, \ldots, k)$ be perfect ideals of $A$. If $A=A_{1}+\cdots+A_{k}$ (direct sum), then $H^{1}(A) \cong H^{1}\left(A_{1}\right)$ $+\cdots+H^{1}\left(A_{k}\right)$ (direct sum).

Proof. Let $c \in \operatorname{Der}(A)$. We denote by $c_{i j}$ the $\operatorname{Hom}\left(A_{i}, A_{j}\right)$-component of $c$. For $x, y \in A_{i}$, we have

$$
\begin{aligned}
c[x, y] & =[c(x), y]+[x, c(y)] \\
& =\sum_{j=1}^{k}\left[c_{i j}(x), y\right]+\sum_{j=1}^{k}\left[x, c_{i j}(y)\right] \\
& =\left[c_{i i}(x), y\right]+\left[x, c_{i i}(y)\right] \in A_{i} .
\end{aligned}
$$

Combined this with $A_{i}=\left[A_{i}, A_{i}\right]$, we obtain $c_{i j}=0$ for $i \neq j$. Put $c_{i i}=c_{i}$. By (3.4), $c_{i}$ induces a derivation of $A_{i}$. Hence $\operatorname{Der}(A)=\operatorname{Der}\left(A_{1}\right)+\cdots+\operatorname{Der}\left(A_{k}\right)$ (direct sum). Our assertion is now evident.

q.e.d.

Lemma 3.7. Let $A$ be an abstract Lie algebra such that $A=A_{1}+A_{2}$ (direct sum) with $A_{1}=\left[A_{1}, A_{1}\right]$. Moreover assume that the center of $A_{1}$ is zero. Then $H^{1}(A) \cong H^{1}\left(A_{1}\right)+H^{1}\left(A_{2}\right)$ (direct sum).

Proof. We can write $c=c_{11}+c_{12}+c_{21}+c_{22}$ by using same notations as Lemma 3.6. Since $A_{1}$ is perfect, we have $c_{12}=0$. Let $x \in A_{1}$ and $y \in A_{2}$. By the equation $0=c[x, y]=[c(x), y]+[x, c(y)]$, we get $\left[x, c_{21}(y)\right]=0$. This 
means that $c_{21}(y) \in\left\{\right.$ center of $\left.A_{1}\right\}$. Since the center of $A_{1}$ is zero, we have $c_{21}=0$. Now it is easy to verify the assertion.

q.e.d.

Combined with Theorem 2.1, we obtain finally the following theorem.

Theorem 3.8. Let $\mathfrak{g}=\sum_{p=-1}^{\infty} \mathfrak{g}_{p}$ be an infinite transitive graded Lie algebra with a semi-simple linear isotropy algebra $\mathrm{g}_{0}$. Then $H^{1}(\mathrm{~g})$ is finite dimensional.

Proof. By Proposition 3.5, $\mathfrak{g}$ has the following form: $\mathfrak{g}=G_{1}+\cdots+G_{m}+$ $G^{\prime}$, where $\operatorname{dim} G^{\prime}<\infty$. Since $G_{1}+\cdots+G_{m}$ is perfect and has no non-trivial center, we have $H^{1}(\mathfrak{g}) \cong H^{1}\left(G_{1}+\cdots+G_{m}\right)+H^{1}\left(G^{\prime}\right)$ by Lemma 3.7. On the other hand, $H^{1}\left(G_{1}+\cdots+G_{m}\right) \cong H^{1}\left(G_{1}\right)+\cdots+H^{1}\left(G_{m}\right)$ by Lemma 3.6, and $\operatorname{dim} H^{1}\left(G_{i}\right)=1$ or 0 for $i=1, \ldots, m$ by Theorem 2.1. (See also Remark 2, and recall that $G_{i}=\mathfrak{g r}\left(L_{\mathfrak{s I}}\left(V_{i}\right)\right)$ or $\mathfrak{g r}\left(L_{\mathfrak{\xi} \mathfrak{p}}\left(V_{i}\right)\right)$.) Thus we obtain $\operatorname{dim} H^{1}(\mathfrak{g})<\infty$.

q.e.d.

\section{§4. The First Cohomology Groups of Infinite Transitive Graded Lie Algebras (II)}

In this section, we assume that the linear isotropy algebra $\mathfrak{g}_{0}$ of $\mathfrak{g}$ $=\sum_{p=-1}^{\infty} \mathfrak{g}_{p}$ contains an element $e$ which satisfies $\left[e, x_{p}\right]=p x_{p}$ for all $x_{p} \in \mathfrak{g}_{p}$. Put $\mathfrak{g}_{-1}=V$. We can write $c(e)=\sum_{p=-1}^{\infty} x_{p}$ with $x_{p} \in \mathfrak{g}_{p}$. For all $v \in V$, we have

$$
[c(e), v]+[e, c(v)]=c[e, v]=-c(v) .
$$

Comparing the $V$-components of this equation, we obtain $\left[x_{0}, v\right]=0$, and hence $x_{0}=0$ by the transitivity condition of $\mathrm{g}$. We now define a new derivation $c^{\prime}$ derived from $c$ by

$$
c^{\prime}=c+\operatorname{ad}\left(\sum_{p \neq 0} \frac{1}{p} x_{p}\right)
$$

It is clear that $c^{\prime}(e)=0$.

Lemma 4.1. $\operatorname{deg} c^{\prime}=0$. (For the definition of "degree" of a derivation, see $§ 1$.

Proof. We must show that $c^{\prime}\left(\mathfrak{g}_{p}\right) \subset \mathfrak{g}_{p}$ for all $p \geqq-1$. Put $c^{\prime}(x)=\sum_{q=-1}^{\infty} y_{q}$ $\left(y_{q} \in \mathfrak{g}_{q}\right)$ for $x \in \mathfrak{g}_{p}$. Then we have

$$
c^{\prime}[e, x]=p c^{\prime}(x)=p \sum_{q=-1}^{\infty} y_{q}=\left[e, c^{\prime}(x)\right]=\sum_{q=-1}^{\infty} q y_{q} .
$$


Hence $y_{q}=0$ for $q \neq p$ and thus $c^{\prime}(x)=y_{p} \in \mathfrak{g}_{p}$.

q.e.d.

Lemma 4.2. If $c^{\prime}=0$ on $V$, then $c^{\prime}=0$ on $\mathrm{g}$.

Proof. For $x \in \mathfrak{g}_{0}$ and $v \in V$, it holds that $\left[c^{\prime}(x), v\right]+\left[x, c^{\prime}(v)\right]=c^{\prime}[x, v]$. By the assumption of $c^{\prime}$, we obtain $\left[c^{\prime}(x), v\right]=0$. Combining $c^{\prime}\left(\mathfrak{g}_{0}\right) \subset \mathfrak{g}_{0}$ with the transitivity of $\mathfrak{g}$, we obtain $c^{\prime}(x)=0$. Repeating this procedure for all $p \geqq 1$, we can also obtain that $c^{\prime}\left(\mathfrak{g}_{p}\right)=0$. Hence $c^{\prime}=0$ on $\mathfrak{g}$.

q.e.d.

Let $[c] \in H^{1}(\mathfrak{g})$ denote an equivalence class of a derivation $c$ of $\mathfrak{g}$. Since $c^{\prime}=c+\operatorname{ad}\left(\sum_{p \neq 0} \frac{1}{p} x_{p}\right)$, we have $[c]=\left[c^{\prime}\right]$. By Lemma 4.1, a restriction of $c^{\prime}$ to $V$ is an element of $\mathfrak{g l}(V)$. We denote this linear mapping by $\left.c^{\prime}\right|_{V}$.

Theorem 4.3. Let $\mathfrak{g}=\sum_{p=-1}^{\infty} \mathfrak{g}_{p}$ be an infinite transitive graded Lie algebra whose linear isotropy algebra $\mathfrak{g}_{0}$ contains an element $e$ which satisfies $\left[e, x_{p}\right]=p x_{p}$ for all $x_{p} \in \mathfrak{g}_{p}$. Then $\operatorname{dim} H^{1}(\mathfrak{g}) \leqq(\operatorname{dim} V)^{2}$.

Proof. Let $c$ be any derivation of $g$. We define a linear mapping $\psi$ : $\operatorname{Der}(\mathfrak{g}) \rightarrow \operatorname{gl}(V)$ by $\psi(c)=\left.c^{\prime}\right|_{V}$. By Lemma 4.2, we obtain that if $c$ is contained in $\operatorname{Ker} \psi$, then $c$ is an inner derivation. Hence our assertion is obvious.

q.e.d.

In case that $\mathfrak{g}$ is derived from $\mathfrak{g}_{0}$, we can get the more precise result. Let $n\left(\mathfrak{g}_{0}\right)$ denote the normalizer of $\mathfrak{g}_{0}$ in $\mathfrak{g l}(V)$. Then we have

Lemma 4.4. Let $\mathfrak{g}$ be a Lie algebra derived from $\mathfrak{g}_{0}$. Then for all $x \in$ $n\left(\mathfrak{g}_{0}\right), \operatorname{ad}(x)$ is a derivation of $\mathfrak{g}$.

Proof. It is sufficient to prove that ad $(x)\left(\mathfrak{g}_{0}^{(p)}\right) \subset \mathfrak{g}_{0}^{(p)}$ for all $p \geqq 1$. Let $z \in \mathrm{g}_{0}^{(1)}$ and $v \in V$. With respect to the bracket operation in $D(V)$, we have

$$
[\operatorname{ad}(x)(z), v]=\operatorname{ad}(x)[z, v]+[z,[v, x]] \in \mathfrak{g}_{0} .
$$

Hence we have ad $(x)(z) \in \mathfrak{g}_{0}^{(1)}$, that is, $\operatorname{ad}(x)\left(\mathfrak{g}_{0}^{(1)}\right) \subset \mathfrak{g}_{0}^{(1)}$. Since $\mathfrak{g}_{0}^{(p+1)}=\left(\mathfrak{g}_{0}^{(p)}\right)^{(1)}$, it can be inductively proved that ad $(x)\left(\mathfrak{g}_{0}^{(p)}\right) \subset \mathfrak{g}_{0}^{(p)}$ for all $p \geqq 1$.

q.e.d.

Theorem 4.5. Let $\mathrm{g}$ be an infinite transitive graded Lie algebra derived from $\mathrm{g}_{0}$. Moreover assume that $\mathrm{g}_{0}$ contains an element $e$ which satisfies $\left[e, x_{p}\right]=p x_{p}$ for all $x_{p} \in \mathfrak{g}_{p}$. Then $H^{1}(\mathrm{~g})$ is isomorphic to $n\left(\mathfrak{g}_{0}\right) / \mathfrak{g}_{0}$.

Proof. By Lemma 4.4, we can define a linear mapping $f: n\left(\mathrm{~g}_{0}\right) \rightarrow H^{1}(\mathfrak{g})$ by $f(x)=[\operatorname{ad}(x)]$. We prove that $f$ is surjective. Let $c$ be any derivation of $\mathfrak{g}$. Recall that $[c]=\left[c^{\prime}\right]$. Since $c^{\prime}$ satisfies $c^{\prime}(V) \subset V$, there exists an element $x$ of $\operatorname{gl}(V)$ such that $c^{\prime}=\operatorname{ad}(x)$ on $V$. Let $v \in V$ and $y \in \mathfrak{g}_{0}$. By the Jacobi identity 
in $D(V)$, we have

$$
\operatorname{ad}(x)[v, y]=[\operatorname{ad}(x)(v), y]+[v, \operatorname{ad}(x)(y)] .
$$

On the other hand, $c^{\prime}$ satisfies

$$
c^{\prime}[v, y]=\left[c^{\prime}(v), y\right]+\left[v, c^{\prime}(y)\right] .
$$

Note that $\operatorname{ad}(x)[v, y]=c^{\prime}[v, y]$ and $\operatorname{ad}(x)(v)=c^{\prime}(v)$. From equations (4.2) and (4.3), it holds that $\left[v,\left(\operatorname{ad}(x)-c^{\prime}\right)(y)\right]=0$. By the transitivity condition of $\mathfrak{g}$, we obtain that $\operatorname{ad}(x)=c^{\prime}$ on $g_{0}$ and hence $x \in n\left(g_{0}\right)$. By Lemma 4.4, ad $(x)$ is a derivation of $\mathfrak{g}$, and $c^{\prime}-\operatorname{ad}(x)$ vanishes on $V$. Now by Lemma 4.2, we clearly have $c^{\prime}=\operatorname{ad}(x)$ on $\mathfrak{g}$. Thus we have proved that $f$ is surjective. Since $\operatorname{Ker} f=\mathfrak{g}_{0}$, we obtain that $H^{1}(\mathfrak{g})$ is isomorphic to $n\left(\mathfrak{g}_{0}\right) / \mathfrak{g}_{0}$.

q.e.d.

\section{§5. Example of Infinite Transitive Graded Lie Algebra $\mathfrak{g}$ with $\operatorname{dim} \mathbb{H}^{1}(\mathfrak{g})=\infty$}

As stated in Introduction, we give an example of $\mathfrak{g}$ such that $H^{1}(\mathfrak{g})$ is of infinite dimension. Note that a derivation $c$ of degree $\leqq-2$ is necessarily an outer derivation. We define a sequence of derived ideals $\mathrm{g}^{(p)}$ of $\mathrm{g}$ inductively by $\mathfrak{g}^{(1)}=[\mathfrak{g}, \mathfrak{g}], \ldots, \mathfrak{g}^{(p)}=\left[\mathfrak{g}^{(p-1)}, \mathfrak{g}^{(p-1)}\right]$. Then we prove

Theorem 5.1. Let $\mathfrak{g}=\sum_{p=-1}^{\infty} \mathfrak{g}_{p}$ be an infinite transitive graded Lie algebra which satisfies $\mathfrak{g}^{(2)}=0$. Then $\operatorname{dim} H^{1}(\mathfrak{g})=\infty$.

Proof. Put $\varphi_{k}=\operatorname{ad}\left(v_{1}\right)$ ad $\left(v_{2}\right) \ldots$ ad $\left(v_{k}\right)$ for $v_{1}, v_{2}, \ldots, v_{k} \in \mathfrak{g}_{-1}$. We show that $\varphi_{k}(k \geqq 1)$ is a derivation of $\mathrm{g}$ by induction. In the case of $k=1, \varphi_{1}=\operatorname{ad}\left(v_{1}\right)$ is an "inner" derivation. Let $k \geqq 1$. Assume that $\varphi_{k}[x, y]=\left[\varphi_{k}(x), y\right]+$ $\left[x, \varphi_{k}(y)\right]$ for any $x, y \in \mathfrak{g}$. Put $\varphi_{k+1}=\varphi_{k}$ ad $\left(v_{k+1}\right)$ for $v_{k+1} \in \mathfrak{g}_{-1}$. Then by the Jacobi identity, we have

$$
\varphi_{k+1}[x, y]=\varphi_{k}\left[v_{k+1},[x, y]\right]=\varphi_{k}\left[\left[v_{k+1}, x\right], y\right]+\varphi_{k}\left[x,\left[v_{k+1}, y\right]\right] .
$$

By the assumption of induction and by $\mathfrak{g}^{(2)}=0$, this element is equal to

$$
\begin{aligned}
\varphi_{k}\left[\left[v_{k+1}, x\right], y\right]+\varphi_{k}\left[x,\left[v_{k+1}, y\right]\right] & =\left[\varphi_{k}\left[v_{k+1}, x\right], y\right]+\left[x, \varphi_{k}\left[v_{k+1}, y\right]\right] \\
& =\left[\varphi_{k+1}(x), y\right]+\left[x, \varphi_{k+1}(y)\right] .
\end{aligned}
$$

Hence $\varphi_{k}$ is a derivation for all $k \geqq 1$. Now if $\varphi_{k}=\operatorname{ad}\left(v_{1}\right) \operatorname{ad}\left(v_{2}\right) \cdots \operatorname{ad}\left(v_{k}\right)=0$ on $\mathfrak{g}$ for all $v_{1}, v_{2}, \ldots, v_{k} \in \mathfrak{g}_{-1}$, we would have $\left[\mathfrak{g}_{-1},\left[\mathfrak{g}_{-1},\left[\ldots,\left[\mathfrak{g}_{-1}, \mathfrak{g}_{k}\right] \cdots\right]=0\right.\right.$. By the transitivity condition of $\mathfrak{g}$, we must have $\mathfrak{g}_{k}=0$. This is a contradiction. 
Thus there exist $v_{1}, v_{2}, \ldots, v_{k} \in \mathrm{g}_{-1}$ for arbitrarily large $k$ such that $\varphi_{k}=\operatorname{ad}\left(v_{1}\right)$. $\operatorname{ad}\left(v_{2}\right) \cdots \operatorname{ad}\left(v_{k}\right) \neq 0$. Since $\operatorname{deg} \varphi_{k}=-k, \varphi_{k}$ is a non-trivial outer derivation of $\mathfrak{g}$, and hence $\operatorname{dim} H^{1}(\mathfrak{g})=\infty$.

q.e.d.

A typical example. Let $\mathfrak{g}_{-1}$ be a two dimensional vector space with a basis $\partial / \partial x, \partial / \partial y$, and let $\mathfrak{g}_{p}$ be a one dimensional vector space with a basis $x^{p+1} \partial / \partial y$ for $p \geqq 0$. Then we have an infinite transitive graded Lie algebra $\mathfrak{g}=\sum_{p=-1}^{\infty} \mathfrak{g}_{p}$, which satisfies $\mathfrak{g}^{(2)}=0$. In this case, $\varphi_{k}=\operatorname{ad}(\partial / \partial x) \cdots \operatorname{ad}(\partial / \partial x)$ are non-trivial derivations of $\mathrm{g}$ for all $k \geqq 1$. Hence $H^{1}(\mathfrak{g})$ is of infinite dimension.

\section{§6. The First Cohomology Groups of Infinite Intransitive}

\section{Lie Algebras $L\left[W^{*}\right]$}

6.1. First we explain a Lie algebra $L\left[W^{*}\right]$ which is a main object in this section. Let $V$ be a finite dimensional vector space with $V=U+W$ (direct sum). We denote by $S\left(W^{*}\right)$ the ring of formal power series over $W$. Let $L$ be an infinite transitive simple Lie algebra over $U$. Both $L$ and $S\left(W^{*}\right)$ are complete topological vector spaces with respect to their natural topology induced by the filtrations. Then a Lie algebra $L\left[W^{*}\right]$ is obtained as a topological completion of $L \otimes S\left(W^{*}\right)$. Since $L\left[W^{*}\right]$ is a perfect Lie algebra, we know that each derivation $c$ of $L\left[W^{*}\right]$ is continuous.

6.2. Let $A$ be an abstract Lie algebra over $\boldsymbol{C}$. Then the commutator ring of $A$, which we denote by $\boldsymbol{C}_{A}$, is defined as follows:

$$
\boldsymbol{C}_{A}=\left\{\rho \in \operatorname{Hom}_{\boldsymbol{C}}(A, A) ; \rho \circ \operatorname{ad}(x)=\operatorname{ad}(x) \circ \rho \text { for all } x \in A\right\} .
$$

In this sub-section we want to determine the commutator rings $\boldsymbol{C}_{L}$ and $\boldsymbol{C}_{L\left[W^{*}\right]}$.

Proposition 6.1. For an infinite transitive simple Lie algebra L, it holds that $\boldsymbol{C}_{L}=\boldsymbol{C}$.

For the proof of Proposition 6.1, we need three lemmas. First we rewrite the some properties of $L$ stated in Section 2 in the following lemma.

Lemma 6.2. (1) $L_{0}=\left[L, L_{1}\right]$, for $L=D(U), L_{31}(U)$ and $L_{3 p}(U)$, (2) $L_{-1}=\left[L, L_{1}\right]$, for $L=L_{\mathrm{ct}}(U)$.

Lemma 6.3. (V. Guillemin [1]). $\quad C_{L}$ is a commutative field which canonically contains the field $\boldsymbol{C}$. 
Proof. For $a \in \boldsymbol{C}$, let $\rho_{a}$ be a mapping such that $x \mapsto a x$ for $x \in L$. Then it is clear that $\rho_{a}$ belongs to $\boldsymbol{C}_{L}$. Through a mapping $a \mapsto \rho_{a}$, we can consider $\mathbb{C}$ is contained in $\boldsymbol{C}_{L}$. Let $\rho$ be a non-zero element of $\mathbb{C}_{L}$. Since $L$ is simple, we have $\rho(L)=L$ and $\operatorname{Ker} \rho=0$. Hence a non-zero $\rho$ has an inverse. Let $\rho_{1}$, $\rho_{2} \in \mathbb{C}_{L}$. It is clear that $\rho_{1} \circ \rho_{2} \in \boldsymbol{C}_{L}$. Now it is sufficient to show that $\rho_{1} \circ \rho_{2}$ $=\rho_{2} \circ \rho_{1}$. For all $x, y \in L$ we have $\rho_{1} \circ \rho_{2}[x, y]=\left[\rho_{1}(x), \rho_{2}(y)\right]=\rho_{2} \circ \rho_{1}[x, y]$. Combining this equation with $L=[L, L]$, we obtain $\rho_{1} \circ \rho_{2}=\rho_{2} \circ \rho_{1}$. $\quad$ q.e.d.

Lemma 6.4. Each $\boldsymbol{C}_{L}$ has a faithful representation as a ring of endomorphisms as follows:

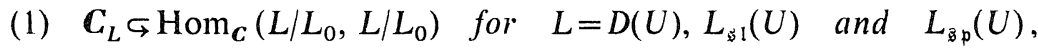

(2) $C_{L} \subseteq \operatorname{Hom}_{C}\left(L / L_{-1}, L / L_{-1}\right)$ for $L=L_{\mathrm{ct}}(U)$.

Proof. (1) Let $\rho$ be an element of $\mathbb{C}_{L}$. Since the filtration $\left\{L_{p}\right\}$ of $L$ satisfies $L_{0}=\left[L, L_{1}\right]$ by Lemma 6.2 , we obtain $\rho\left(L_{0}\right) \subset L_{0}$. Hence a linear mapping $\rho \mapsto \bar{\rho} \in \operatorname{Hom}_{\boldsymbol{C}}\left(L / L_{0}, L / L_{0}\right)$ is naturally induced. Assume $\bar{\rho}=0$. Then $\rho(L)$ is an ideal of $L$ contained in $L_{0}$, and hence $\rho(L)=0$. Thus a linear mapping $\rho \rightarrow \bar{\rho}$ is faithful. The assertion (2) is proved by the same argument as (1).

q.e.d.

Proof of Proposition 6.1. First let $L$ be an infinite irreducible transitive Lie algebra. Then by Remark 1 in Section 2, the linear isotropy algebra $g_{0}$ of $L$ is considered as a Lie subalgebra of $\mathrm{gl}(U)$. Recall that $\mathrm{g}_{0}$ of $L=D(U)$,

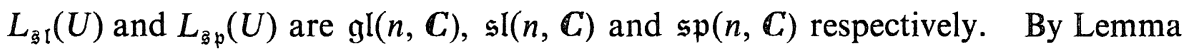
6.3 and Lemma $6.4, C_{L}$ can be regarded as an abelian Lie subalgebra of $\mathfrak{g l}(U)$. We will show that $\boldsymbol{C}_{L}$ is contained in the centralizer of $\mathfrak{g}_{0}$ in $\operatorname{gl}(U)$. Let $\rho \in \mathbb{C}_{L}$, $x \in \mathfrak{g}_{0}$ and $u \in U$. Then in $D(U)$ we clearly have

$$
[[\rho, x], u]=[\rho,[x, u]]-[x,[\rho, u]]=(\rho \circ \operatorname{ad}(x)-\operatorname{ad}(x) \circ \rho)(u)=0 .
$$

Since $[\rho, x] \in \mathfrak{g}_{0}$ and $L$ is transitive, we obtain $[\rho, x]=0$, and hence $\left[\mathbb{C}_{L}, \mathfrak{g}_{0}\right]=0$. Put $\tilde{\mathrm{g}}_{0}=\mathrm{g}_{0}+\mathbb{C}_{L}$. Then $\tilde{\mathrm{g}}_{0}$ yields a Lie subalgebra of $\mathrm{gl}(U)$ and $\mathbb{C}_{L}$ is contained in the center of $\tilde{\mathfrak{g}}_{0}$. Since $\mathfrak{g}_{0}$ acts irreducibly on $U, \tilde{\mathfrak{g}}_{0}$ also acts irreducibly on $U$. Note that $\tilde{g}_{0}$ is of infinite type. By the classification theorem of Lie algebras of infinite type ([2] or [4]), $\tilde{\mathfrak{g}}_{0}$ must be equal to $\mathfrak{g l}(U)$ or $\operatorname{csp}(U)$. Thus we have $\boldsymbol{C}_{L}=\mathbf{C}$.

Next let $L=L_{\mathrm{ct}}(U)$. Put $L / L_{-1}=U^{\prime}$. Then $U^{\prime}$ is a one dimensional subspace of $\mathrm{gl}\left(U^{\prime}\right)$, which contains $\mathbb{C}$. Hence $\mathbb{C}_{L}=\mathbb{C}$.

q.e.d. 
Using Proposition 6.1, we can verify the following proposition originally proved by V. Guillemin [1].

Proposition 6.5. The commutator ring of $L\left[W^{*}\right]$, i.e., $\mathbb{C}_{L\left[W^{*}\right]}$, is isomorphic to $S\left(W^{*}\right)$.

Outline of proof. We will regard $L$ as imbedded in $L\left[W^{*}\right]$. Let $\rho$ be an element of $\mathbb{C}_{L\left[W^{*}\right]}$. We will denote by $\left\{f^{\alpha}\right\}$ the monomial basis in $S\left(W^{*}\right)$. If $x \in L$, then we can write

$$
\rho(x)=\sum_{\alpha=0}^{\infty} \rho_{\alpha}(x) f^{\alpha}, \quad \rho_{\alpha}(x) \in L
$$

where $\rho_{\alpha}$ depends linearly on $x$. Since $\rho$ is an element of $\mathbb{C}_{L\left[W^{*}\right]}$, we clearly obtain $\rho_{\alpha} \in \boldsymbol{C}_{L}$. By Proposition 6.1, $\rho_{\alpha}$ is an element of $\boldsymbol{C}$. Hence we can write

$$
\rho(x)=x \otimes \prod_{\alpha=0}^{\infty} \rho_{\alpha} f^{\alpha}, \quad \text { for all } \quad x \in L .
$$

Since $L$ is simple, we have $\left[L, L\left[W^{*}\right]\right]=L\left[W^{*}\right]$. Hence if $\rho \in \mathbb{C}_{L\left[W^{*}\right]}$, it is determined completely by its restriction to $L$. The isomorphism between $\mathbb{C}_{L\left[W^{*}\right]}$ and $S\left(W^{*}\right)$ is given by $\rho \mapsto \prod_{\alpha=0}^{\infty} \rho_{\alpha} f^{\alpha}$. This completes the proof. $\quad$ q.e.d.

By Proposition 6.5, $\operatorname{Der}\left(\mathbb{C}_{L\left[W^{*}\right]}\right)$ is identified with $\operatorname{Der}\left(S\left(W^{*}\right)\right)$. Now we have a homomorphism: $l: \operatorname{Der}\left(S\left(W^{*}\right)\right) \rightarrow \operatorname{Der}\left(L\left[W^{*}\right]\right)$. Let $X \in \operatorname{Der}\left(L\left[W^{*}\right]\right)$ and $\rho \in \boldsymbol{C}_{L\left[W^{*}\right]}$. Then $X \circ \rho-\rho \circ X$ is an element of $\boldsymbol{C}_{L\left[W^{*}\right]}$. We denote this element of $\mathbb{C}_{L\left[W^{*}\right]}$ by $L_{X} \rho$. By an easy consideration, the mapping $X \mapsto L_{X}$ is a homomorphism of $\operatorname{Der}\left(L\left[W^{*}\right]\right)$ into $\operatorname{Der}\left(\mathbb{C}_{L\left[W^{*}\right]}\right)=\operatorname{Der}\left(S\left(W^{*}\right)\right)$. Hence there is a natural homomorphism

$$
L: \operatorname{Der}\left(L\left[W^{*}\right]\right) \longrightarrow \operatorname{Der}\left(S\left(W^{*}\right)\right) .
$$

It is easy to see that $L \circ l=$ identity, which implies that a homomorphism $L$ is surjective. Since any elements of the kernel of $L$ are $S\left(W^{*}\right)$-linear mappings, the kernel of $L$ is identified with the set of all mappings $c: L \rightarrow L\left[W^{*}\right]$ satisfying the identity

$$
c[x, y]=[c(x), y]+[x, c(y)] \quad \text { for all } x, y \in L .
$$

We denote this set by $\operatorname{Der}\left(L, L\left[W^{*}\right]\right)$.

Summarizing the above remarks, we have

Proposition 6.6 (V. Guillemin [1]). There is a split exact sequence of Lie algebras: 


$$
0 \longrightarrow \operatorname{Der}\left(L, L\left[W^{*}\right]\right) \longrightarrow \operatorname{Der}\left(L\left[W^{*}\right]\right) \underset{\longleftarrow_{l}^{L}}{\longrightarrow} \operatorname{Der}\left(S\left(W^{*}\right)\right) \longrightarrow 0 .
$$

6.3. In this sub-section, we will determine the first cohomology group $H^{1}\left(L\left[W^{*}\right]\right)$. By Proposition 6.6, we have a natural isomorphism:

$$
\operatorname{Der}\left(L\left[W^{*}\right]\right) \cong \operatorname{Der}\left(L, L\left[W^{*}\right]\right)+\operatorname{Der}\left(S\left(W^{*}\right)\right) \quad \text { (direct sum) } .
$$

The space $\operatorname{Der}\left(S\left(W^{*}\right)\right)$ is canonically identified with $D(W)$, the Lie algebra of all formal vector fields over $W$. Hence it suffices to determine $\operatorname{Der}\left(L, L\left[W^{*}\right]\right)$ for calculating $\operatorname{Der}\left(L\left[W^{*}\right]\right)$.

Let $x \in L$ and $c \in \operatorname{Der}\left(L, L\left[W^{*}\right]\right)$. We denote by $f^{\alpha}$ the basis of $S\left(W^{*}\right)$ consisting of monomials. Then we can write:

$$
c(x)=\prod_{\alpha=0}^{\infty} x_{\alpha} \otimes f^{\alpha}, \quad x_{\alpha} \in L .
$$

Put $x_{\alpha}=c_{\alpha}(x)$. Then $c_{\alpha}$ is a linear mapping of $L$ into itself. For $x, y \in L$, we have

$$
\begin{aligned}
c[x, y] & =\prod_{\alpha=0}^{\infty} c_{\alpha}[x, y] \otimes f^{\alpha}=[c(x), y]+[x, c(y)] \\
& =\left[\prod_{\alpha=0}^{\infty} c_{\alpha}(x) \otimes f^{\alpha}, y\right]+\left[x, \prod_{\alpha=0}^{\infty} c_{\alpha}(y) \otimes f^{\alpha}\right] \\
& =\prod_{\alpha=0}^{\infty}\left(\left[c_{\alpha}(x), y\right]+\left[x, c_{\alpha}(y)\right]\right) \otimes f^{\alpha} .
\end{aligned}
$$

Hence $c_{\alpha}[x, y]=\left[c_{\alpha}(x), y\right]+\left[x, c_{\alpha}(y)\right]$, which implies that $c_{\alpha}$ is an element of $\operatorname{Der}(L)$. By Theorem 2.1, there exists a unique element $z_{\alpha}$ of $L$ (resp. $L+\mathbb{C} e$ ) such that $c_{\alpha}=\operatorname{ad}\left(z_{\alpha}\right)$ for $L=D(U)$ or $L_{\mathrm{ct}}(U)$ (resp. $L=L_{\mathfrak{\xi} \mathrm{r}}(U)$ or $L_{\mathfrak{\xi} p}(U)$ ). Thus we have $c=\operatorname{ad}\left(\prod_{\alpha=0}^{\infty} z_{\alpha} \otimes f^{\alpha}\right)$. Here the symbol $e$ denotes a unit matrix, i.e. a basis of one dimensional center of $\mathfrak{g l}(U)$. Now we easily obtain the following isomorphism:

$\operatorname{Der}\left(L\left[W^{*}\right]\right) \cong\left\{\begin{array}{l}L\left[W^{*}\right]+D(W) \quad \text { for } L=D(U) \text { or } L_{\mathfrak{c t}}(U) \\ \left(L\left[W^{*}\right]+S\left(W^{*}\right) \otimes e\right)+D(W) \text { for } L=L_{\mathfrak{\jmath} \mathfrak{l}}(U) \text { or } L_{\mathfrak{s} \mathfrak{p}}(U) .\end{array}\right.$

Since $L\left[W^{*}\right]$ has no non-trivial center, the space ad $\left(L\left[W^{*}\right]\right)$ of all inner derivations of $L\left[W^{*}\right]$ is naturally isomorphic to $L\left[W^{*}\right]$.

Summarizing the above results, we have proved:

Theorem 6.7. Let $D(W)$ be a Lie algebra of all formal vector fields over $W$ and let $e$ be a basis of one dimensional center of $\mathfrak{g l}(U)$. Then we have the following isomorphism: 
$H^{1}\left(L\left[W^{*}\right]\right) \cong\left\{\begin{array}{l}D(W) \quad \text { for } L=D(U) \text { or } L_{\mathrm{ct}}(U) \\ D(W)+S\left(W^{*}\right) \otimes e \text { for } L=L_{\vec{p} 1}(U) \text { or } L_{\mathfrak{p} p}(U) .\end{array}\right.$

\section{References}

[1] Guillemin, V., A Jordan-Hölder decomposition for certain class of infinite dimensional Lie algebras, J. Differential Geometry, 2 (1968), 313-345.

[2] Guillemin, V., Quillen, D. and Sternberg, S., The classification of the complex primitive infinite pseudogroups, Proc. Nat. Acad. Sci. U. S. A., 55 (1966), 687-690.

[3] Kanie, Y., Some Lie algebras of vector fields and their derivations; Case of partially classical type, (to appear in Nagoya Math. $J$.).

[4] Kobayashi, S. and Nagano, T., On filtered Lie algebras and geometric structures, III and IV, J. Math. Mech., 14 (1965), 679-706 and 15 (1966), 163-175.

[5] Morimoto, T., The derivation algebras of the classical infinite Lie algebras, J. Math. Kyoto Univ., 16 (1976), 1-24.

[6] -, On the intransitive Lie algebras whose transitive parts are infinite and primitive, J. Math. Soc. Japan, 29 (1977), 35-65. 